\title{
ata34
}

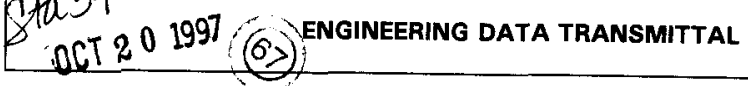

\begin{tabular}{|l|l|}
\hline $\begin{array}{l}\text { 2. To: (Receiving Organization) } \\
\text { LMHC/TWRS }\end{array}$ & $\begin{array}{l}\text { 3. From: (Originating Organization) } \\
\text { Tank Farm Upgrade Projects }\end{array}$ \\
\hline 5ast Tank Farms & $\begin{array}{l}\text { 6. Design Authority/ Design Agent/Cog. } \\
\text { Projog./Dept./Div.: }\end{array}$ \\
\hline Droject W-314 & E. BOWERS \\
\hline
\end{tabular}

8. Originator Remarks:

Initial release of the Acceptance for Beneficial Use for the MASTER PUMP SHUTDOWN Upgrade

11. Receiver Remarks: 11A. Design Basel ine Document? [] res [X] No
4. Related EDT No.:
$N / A$
7. Purchase Order No.:
$\mathrm{N} / \mathrm{A}$

9. Equip./Component No,:

N/A

10. System/Bldg./Facility: $\mathrm{N} / \mathrm{A}$

12. Major Assm. Dwg. No.: N/A

13. Permit/Permit Application No.: $N / A$

14. Required Response Date: $\mathrm{N} / \mathrm{A}$

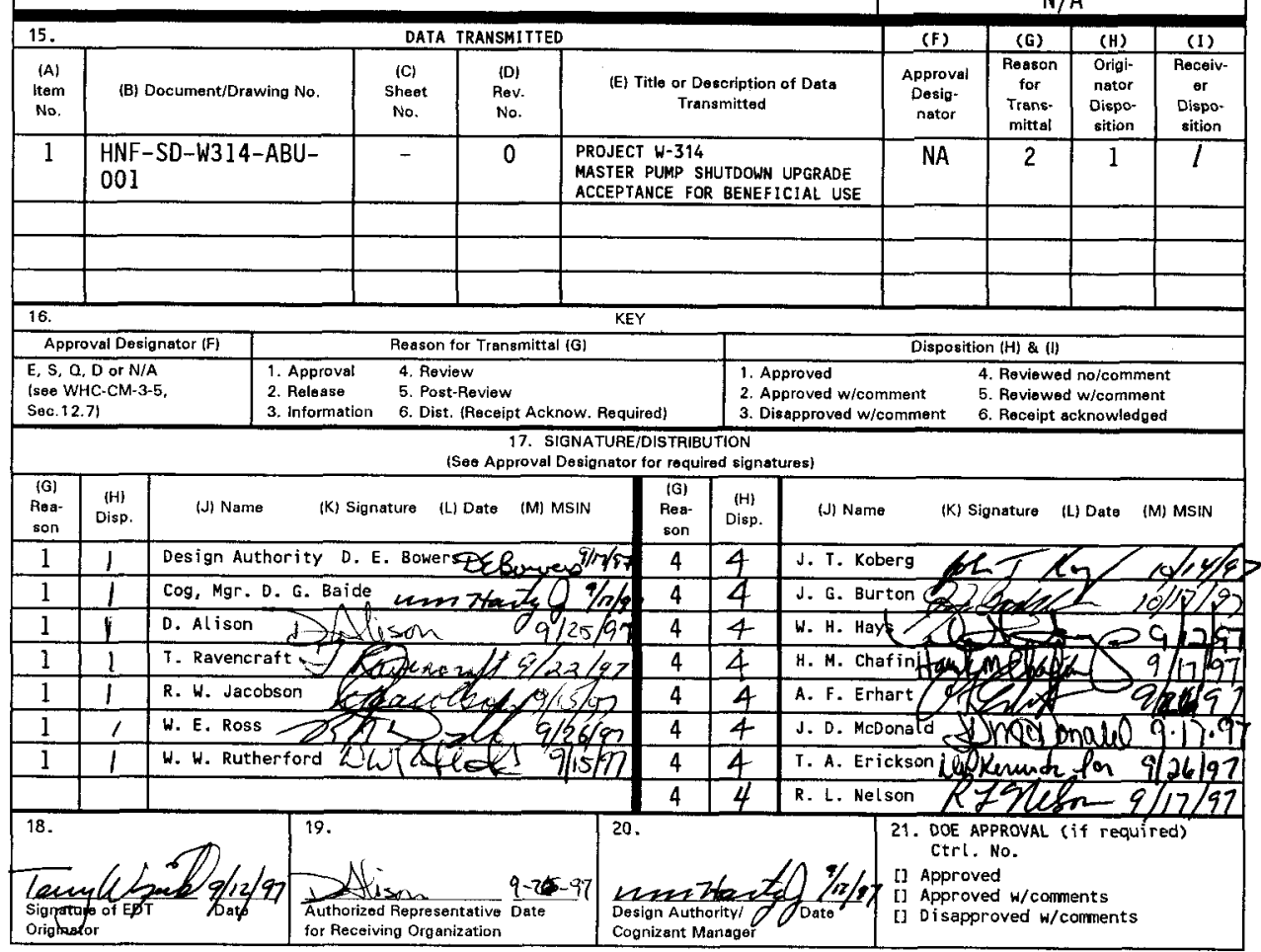




\section{PROJECT W-314 \\ MASTER PUMP SHUTDOWN UPGRADE Acceptance For Beneficial Use}

Terry Warnick

Fluor Daniel Northwest, Richland, WA 99352

U.S. Department of Energy Contract DE-AC06-96RL13200

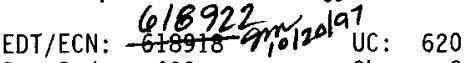

Org Code: 408

Charge Code: P4LVOO

B\&R Code: EW3130010 Tota1 Pages: 13

Key Words: MASTER PUMP SHUTDOWN, ABU

Abstract: N/A

TRADEMARK DISCLAIMER. Reference herein to any specific commercial product, process, or service by trade name, trademark, manufacturer, or otherwise, does not necessarily constitute or imply its endorsement, recommendation, or favoring by the United States Government or any agency thereof or its contractors or subcontractors.

Printed in the United States of America. To obtain copies of this document, contact: Document Control Services, P.0. Box 950, Maitstop H6-08, Richland WA 99352, Phone (509) 372-2420; Fax (509) 376-4989.
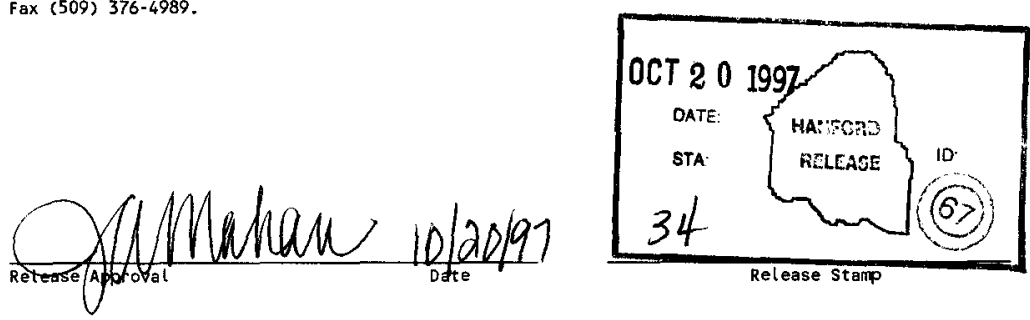

Approved for Public Release 


\begin{tabular}{|c|c|c|c|}
\hline \multicolumn{4}{|c|}{ DOQUMENTATION REQULRED for ACOPPANCE GOR BENEFICLAL USE } \\
\hline DESCRIPTION & RESPONSIBILITY & DESCRIPTION & RESPONSIBILITY \\
\hline ENGINEERING & & ENGINEERING (cont'd) & \\
\hline [ ] Engineering Task Plan - N/A & & [X] Construction Specification & Rickenbach \\
\hline$[X]$ Activity Schedule & Rutherford & [ ] Essential Material Specification - N/A & \\
\hline$[\mathrm{X}]$ USQ Screening & Leach & [ ] Installation Work Plan - N/A & \\
\hline [ ] Final Safety Analysis Report - AB Amendment - N/A & & {$[\mathrm{X}]$ Final Design Drawings } & Rickenbach \\
\hline [ ] lnterim Safety Basis - $A B(B l O)$ Amendment - N/A & & [ ] Installation Drawings - N/A & \\
\hline 1 ] Safety Assessment $-\mathrm{N} / \mathrm{A}$ & & {$[\mathrm{X}]$ As-Buitt Drawings $\mathrm{H}-2$} & Rickenbach \\
\hline$[\mathrm{X}]$ Safety Equipment List & Homan & [X] PCP Consiruction Inspection Records & Miller \\
\hline [ ] Operational Safety Document(s) - N/A & & {$[\mathrm{X}]$ IEFD Drawings - (P \& ID) } & Rickenbach \\
\hline$[X]$ Design Criteria (Project Development Specification (PDS)) & Bowers & {$[\mathrm{X}]$ Systems Drawings H-14 (BIO, "Eşsential") } & Rickenbach \\
\hline$[\mathrm{X}]$ Requirements Verification Report for PDS & Rickenbach/Hays & {$[X]$ Drawing Tree/Index } & Rickenbach \\
\hline [ ] System Design Description-N/A & & [X] As-Built Project Generated Drawings/ECNs & Rickenbach \\
\hline$[\mathrm{X}]$ Project Design Concept (PDC) & Rickenbach & {$[\mathrm{X}]$ Void Superseded Drawings } & Nelson \\
\hline$[\mathrm{X}]$ Test Plan/Specifications (TEP) & Hays & [X] Master Equipment List Update & Nelson \\
\hline [X] Acceptance Test Procedures & Rickenbach & ADP (all compuler activities are on MPS ABU) & \\
\hline [X] Acceptance Test Report & Hays & {$[\mathrm{X}]$ Software Configuration Management Plan } & Rickenbach \\
\hline$[X]$ Operational Test Procedures & Hays & {$[X]$ Systems Requirements Spec } & Rickenbach \\
\hline$[\mathrm{X}]$ Operational Test Report & Hays & {$[X]$ Software Design Description } & Rickenbach \\
\hline [X] Environmental Impact Statement (TWRS) & Boes & {$[X]$ Software Validation/Verification } & Rickenbach \\
\hline [ ] Environmental Report - N/A & & TRAINING & \\
\hline I ] Environmental Permit - N/A & & {$[\mathrm{X}]$ Training Plan } & Morris \\
\hline I ] Hazardous Waste Disposal Plan/Procedures - N/A & & {$[X]$ Training Manuals/Certification Package } & Morris \\
\hline [ ] Solid Waste Disposal Plan/Procedure - N/A & & {$[\mathrm{X}]$ Training to Operating Crews } & Morris \\
\hline$[\mathrm{X}]$ Notice of Constnuction/Excavation & Boes & {$[\mathrm{X}]$ Training to Maintenance Crews } & Morris \\
\hline$[\mathrm{X}]$ Stress/Seismic Analysis & Rickenbach & {$[X]$ Training Mock-Up } & Hays \\
\hline [ ] Stress/Design Report - N/A & & QUALITY ASSURANCE & Talys \\
\hline I ] Design Specification Report - N/A & & {$[\mathrm{X}]$ Inspection Plans } & Chafin \\
\hline ( ) Design Specifications/Report - N/A & & {$[X]$ QAPP } & Chafin \\
\hline I I Equipment Specifications - N/A & & [ ] QAPjP - N/A & \\
\hline [X] Procurenent Specifications & Rickenbach & & \\
\hline
\end{tabular}


Program/Project Title: Project W-3 14, Tank Farm Restoration and Safe Operation, Phase 1

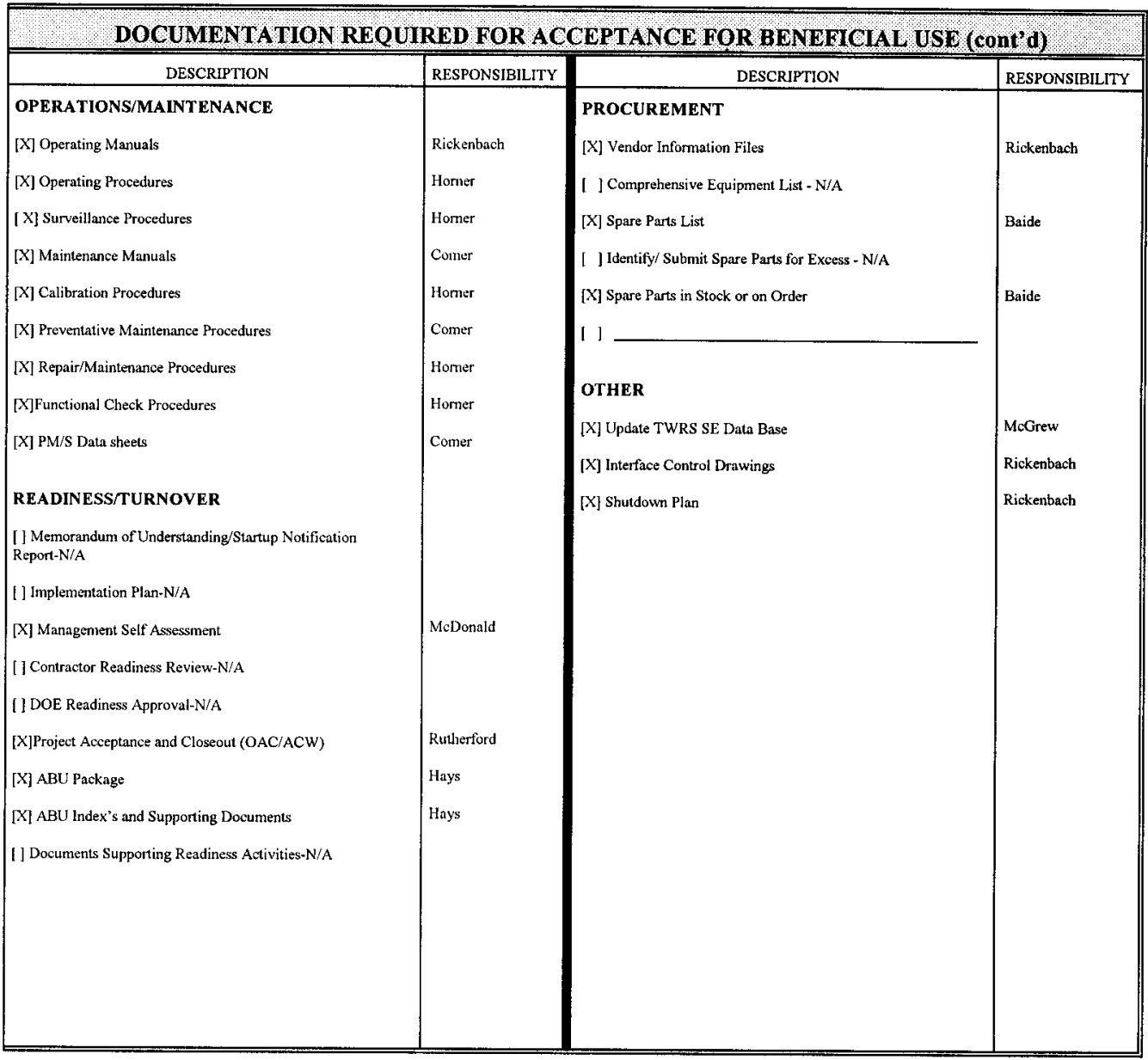




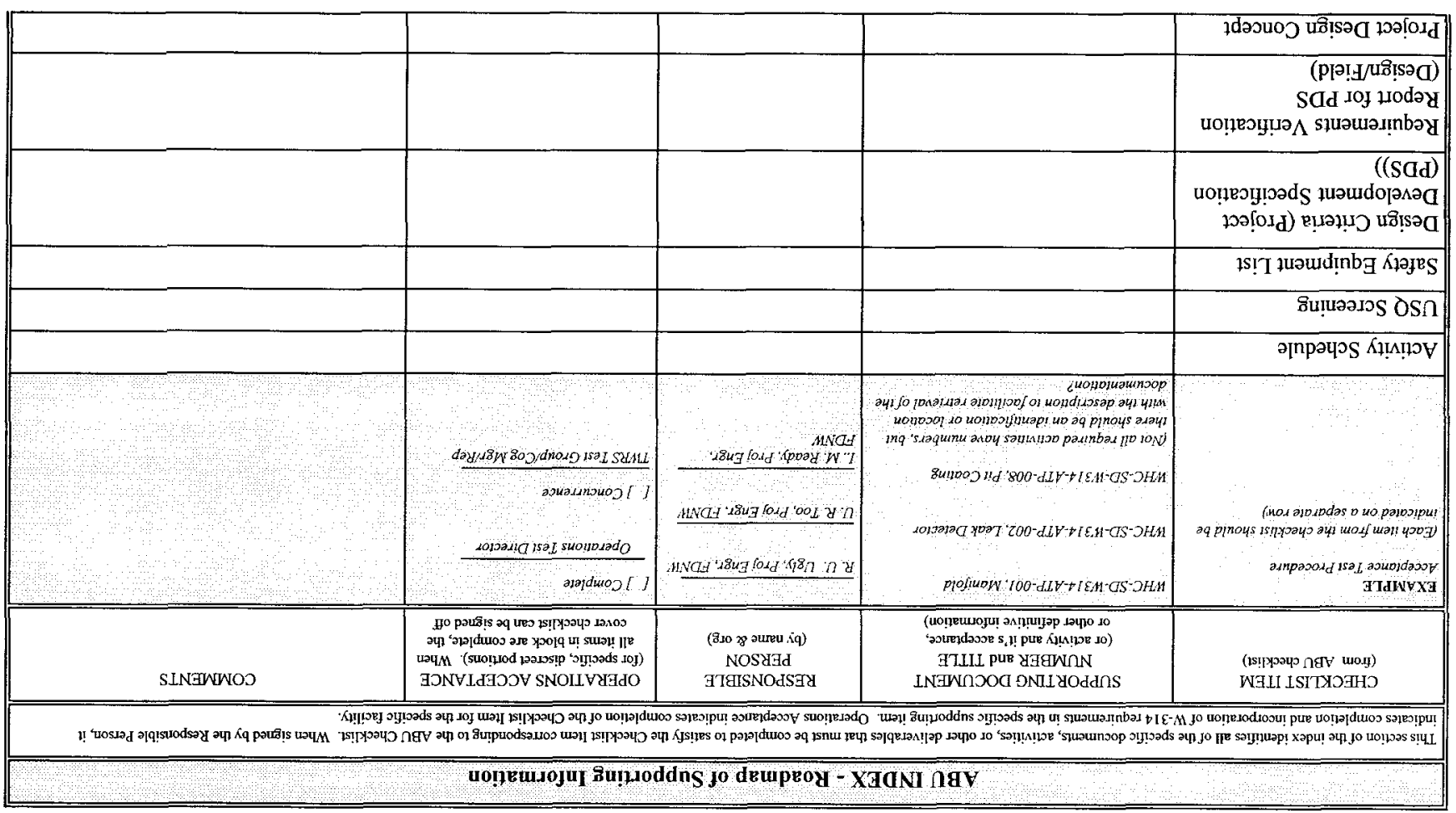


Program/Project Title: Project W-314, Tank Farm Restoration and Safe Operation, Phase 1

Component/System: Master Pump Shutdown Upgrade September 12, 1997

\section{ABU INDEX - Roadmap of Supporting Information}

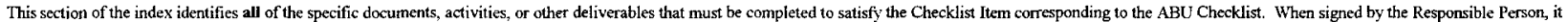
indicates completion and incorporation of W-314 requirements in the specific supporting item. Operations Acceptance indicates completion of the Checklist ltem for the specific facility.

\begin{tabular}{|c|c|c|c|c|}
\hline $\begin{array}{l}\text { CHECKLIST ITEM } \\
\text { (from ABU checklist) }\end{array}$ & $\begin{array}{l}\text { SUPPORTING DOCUMENT } \\
\text { NUMBER and TITLE } \\
\text { (or activity and it's acceptance, } \\
\text { or other definitive information) }\end{array}$ & $\begin{array}{c}\text { RESPONSIBLE } \\
\text { PERSON } \\
\text { (by name \& org) }\end{array}$ & $\begin{array}{l}\text { OPERATIONS ACCEPTANCE } \\
\text { (for specific, discreet portions). When } \\
\text { all items in block are complete, the } \\
\text { cover checklist can be signed off }\end{array}$ & COMMENTS \\
\hline \multicolumn{5}{|l|}{$\begin{array}{l}\text { Test Plans/Specifications } \\
\text { (TEP) }\end{array}$} \\
\hline \multicolumn{5}{|l|}{$\begin{array}{l}\text { Acceptance Test } \\
\text { Procedures }\end{array}$} \\
\hline \multicolumn{5}{|l|}{ Acceptance Test Report } \\
\hline \multicolumn{5}{|l|}{$\begin{array}{l}\text { Operational Test } \\
\text { Procedures }\end{array}$} \\
\hline \multicolumn{5}{|l|}{ Operational Test Report } \\
\hline \multicolumn{5}{|l|}{$\begin{array}{l}\text { Environmental Impact } \\
\text { Statement (TWRS) }\end{array}$} \\
\hline \multicolumn{5}{|l|}{$\begin{array}{l}\text { Notice of } \\
\text { Construction/Excavation }\end{array}$} \\
\hline \multicolumn{5}{|l|}{ Stress/Seismic Analysis } \\
\hline \multicolumn{5}{|l|}{ Procurement Specification } \\
\hline \multicolumn{5}{|c|}{ Constructions Specification } \\
\hline \multicolumn{5}{|l|}{ Final Design Drawings } \\
\hline As-Build Drawings $\mathrm{H}-2$ & & & & \\
\hline
\end{tabular}

HNF-SD-W314-ABU-001, Rev 0

Page 4 of 12 
Program/Project Title: Project W-314, Tank Farm Restoration and Safe Operation, Phase 1

Component/System: Master Pump Shutdown Upgrade September 12, 1997

\section{ABU INDEX - Roadmap of Supporting Information}

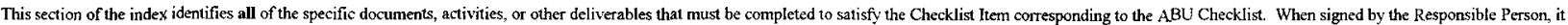
indicates completion and incorporation of W-314 requirements in the specific supporting item. Operations Acceptance indicates completion of the Checklist Item for the specific facility

\begin{tabular}{|c|c|c|c|c|}
\hline $\begin{array}{l}\text { CHECKLIST ITEM } \\
\text { (from ABU checklist) }\end{array}$ & $\begin{array}{l}\text { SUPPORTING DOCUMENT } \\
\text { NUMBER and TITLE } \\
\text { (or activity and it's acceptance, } \\
\text { or other definitive information) } \\
\end{array}$ & $\begin{array}{c}\text { RESPONSIBLE } \\
\text { PERSON } \\
\text { (by name \& org) }\end{array}$ & $\begin{array}{l}\text { OPERATIONS ACCEPTANCE } \\
\text { (for specific, discreet portions). Wher) } \\
\text { all items in block are complete, the } \\
\text { cover checklist can be signed off }\end{array}$ & COMMENTS \\
\hline \multicolumn{5}{|l|}{$\begin{array}{l}\text { PCP Construction } \\
\text { Inspection Records }\end{array}$} \\
\hline \multicolumn{5}{|c|}{ IEFD Drawings- (P\&ID) } \\
\hline \multicolumn{5}{|l|}{$\begin{array}{l}\text { Systems Drawings H-14 } \\
\text { (BIO, ESSENTIAL) }\end{array}$} \\
\hline \multicolumn{5}{|l|}{ Drawing Tree Index } \\
\hline \multicolumn{5}{|c|}{$\begin{array}{l}\text { As-Build Project Generated } \\
\text { Drawings/ECNs }\end{array}$} \\
\hline \multicolumn{5}{|c|}{ Void Superseded Drawings } \\
\hline \multicolumn{5}{|l|}{$\begin{array}{l}\text { Master Equipment List } \\
\text { Update }\end{array}$} \\
\hline \multicolumn{5}{|l|}{$\begin{array}{l}\text { Software Configuration } \\
\text { Management Plan }\end{array}$} \\
\hline \multicolumn{5}{|l|}{$\begin{array}{l}\text { Systems Requirements } \\
\text { Specification }\end{array}$} \\
\hline \multicolumn{5}{|l|}{$\begin{array}{l}\text { Software Design } \\
\text { Description }\end{array}$} \\
\hline $\begin{array}{l}\text { Software Validation/ } \\
\text { Verification }\end{array}$ & & & & \\
\hline
\end{tabular}

HNF-SD-W3 14-ABU-001, Rev 0

Page 5 of 12 
Program/Project Title: Project W-314, Tank Farm Restoration and Safe Operation, Phase 1

\section{Component/System: Master Pump Shutdown Upgrade September 12, 1997}

\section{ABU INDEX - Roadmap of Supporting Information}

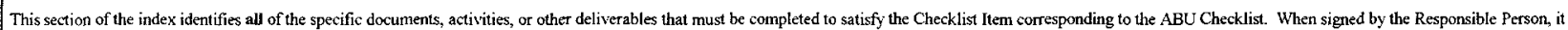
indicates completion and incorporation of W-314 requirements in the specific supporting itern. Operations Acceptance indicates completion of the Checklist Item for the specific facility.

\begin{tabular}{|c|c|c|c|c|}
\hline $\begin{array}{l}\text { CHECKLIST ITEM } \\
\text { (from ABU checklist) }\end{array}$ & $\begin{array}{l}\text { SUPPORTING DOCUMENT } \\
\text { NUMBER and TITLE } \\
\text { (or activity and it's acceptance, } \\
\text { or other definitive information) }\end{array}$ & $\begin{array}{c}\text { RESPONSIBLE } \\
\text { PERSON } \\
\text { (by name \& org) }\end{array}$ & $\begin{array}{l}\text { OPERATIONS ACCEPTANCE } \\
\text { (for specific, discreet portions). When } \\
\text { all items in block are complete, the } \\
\text { cover checklist can be signed off }\end{array}$ & COMMENTS \\
\hline \multicolumn{5}{|l|}{ Training Plan } \\
\hline \multicolumn{5}{|l|}{$\begin{array}{l}\text { Training } \\
\text { Manuals/Certification } \\
\text { Package }\end{array}$} \\
\hline \multicolumn{5}{|l|}{$\begin{array}{l}\text { Training to Operating } \\
\text { Crews }\end{array}$} \\
\hline \multicolumn{5}{|l|}{$\begin{array}{l}\text { Training to Maintenance } \\
\text { Crews }\end{array}$} \\
\hline \multicolumn{5}{|l|}{ Training Mock-Up } \\
\hline \multicolumn{5}{|l|}{ Inspection Plans } \\
\hline \multicolumn{5}{|l|}{ QAPP } \\
\hline \multicolumn{5}{|l|}{ Operating Manuals } \\
\hline \multicolumn{5}{|l|}{ Operating Procedures } \\
\hline \multicolumn{5}{|l|}{ Surveillance Procedures } \\
\hline \multicolumn{5}{|l|}{ Maintenance Manuals } \\
\hline Calibration Procedures & & & & \\
\hline
\end{tabular}

HNF-SD-W314-ABU-001, Rev 0

Page 6 of 12 
Program/Project Title: Project W-314. Tank Farm Restoration and Safe Operation, Phase 1

Component/System: Master Pump Shutdown Upgrade September 22, 1997

\section{ABU INDEX - Roadmap of Supporting Information}

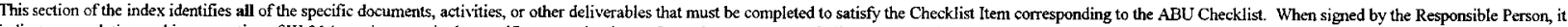
indicates completion and incorporation of W-314 requirements in the specific supporting item. Operations Acceptance indicates completion of the Checklist Item for the specific facility.

\begin{tabular}{|c|c|c|c|c|}
\hline $\begin{array}{l}\text { CHECKLIST ITEM } \\
\text { (from ABU checklist) }\end{array}$ & $\begin{array}{l}\text { SUPPORTING DOCUMENT } \\
\text { NUMBER and TITLE } \\
\text { (or activity and it's acceptance, } \\
\text { or other definitive information) } \\
\end{array}$ & $\begin{array}{l}\text { RESPONSIBLE } \\
\text { PERSON } \\
\text { (by name \& org) }\end{array}$ & $\begin{array}{l}\text { OPERATIONS ACCEPTANCE } \\
\text { (for specific, discreet portions). When } \\
\text { all items in block are complete, the } \\
\text { cover checklist can be signed off }\end{array}$ & COMMENTS \\
\hline \multicolumn{5}{|l|}{$\begin{array}{l}\text { Preventative Maintenance } \\
\text { Procedures }\end{array}$} \\
\hline \multicolumn{5}{|l|}{$\begin{array}{l}\text { Repair/Maintenance } \\
\text { Procedures }\end{array}$} \\
\hline \multicolumn{5}{|l|}{$\begin{array}{l}\text { Functional Check } \\
\text { Procedures }\end{array}$} \\
\hline \multicolumn{5}{|l|}{ PM/S Data sheets } \\
\hline \multicolumn{5}{|l|}{$\begin{array}{l}\text { Management Self } \\
\text { Assessment }\end{array}$} \\
\hline $\begin{array}{l}\text { Project Acceptance and } \\
\text { Closeout (OAC/ACW) }\end{array}$ & $\begin{array}{l}\text { ACW-AN MPS Installation } \\
\text { ACW-242-A MPS Installation } \\
\text { ACW-SY MPS Installation } \\
\text { ACW-AP MPS Installation } \\
\text { ACW-244-S MPS Installation } \\
\text { ACW-AY/AZ MPS Installation } \\
\text { ACW-A MPS Installation } \\
\text { ACW-244-A MPS Installation } \\
\text { ACW-278-WA MPS Installation } \\
\text { ACW-272-AW MPS Installation } \\
\text { ACW-AW MPS Installation } \\
\end{array}$ & & & \\
\hline ABU Package & & & & \\
\hline
\end{tabular}

HNF-SD-W3 14-ABU-001, Rev 0

Page 7 of 12 
Program/Project Title: Project W-314, Tank Farm Restoration and Safe Operation, Phase 1

Component/System: Master Pump Shutdown Upgrade September 22, 1997

\section{ABU INDEX - Roadmap of Supporting Information}

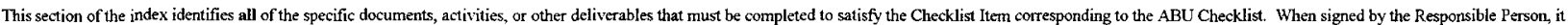
indicates completion and incorporation of W.314 requirements in the specific supporting item. Operations Acceptance indicates completion of the Checklist Item for the specific facility.

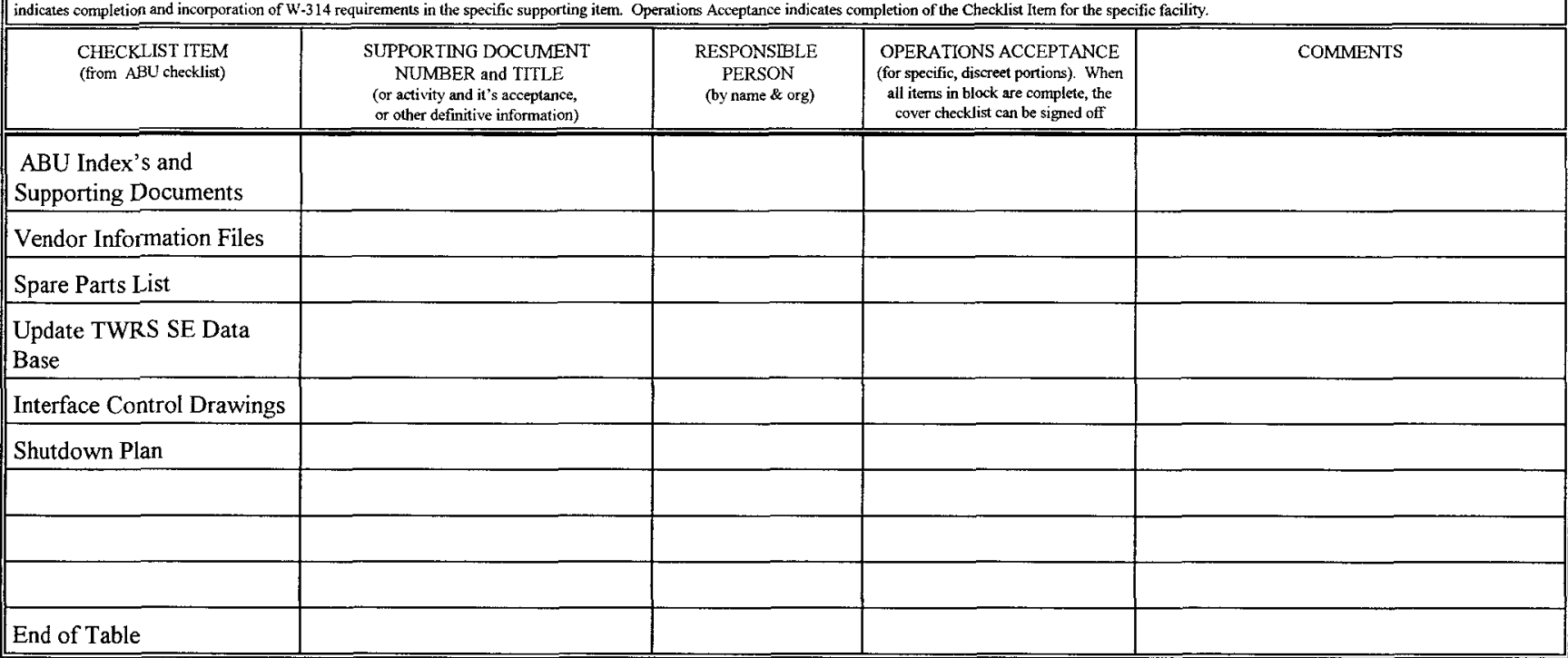


Program/Project Title: Project W-314, Tank Farm Restoration and Safe Operation, Phase 1 Component/System: Master Pump Shutdown Upgrade

September 22, 1997

\section{ABU INDEX - Acceptance Documentation}

This section of the index identiffes Operations acceptance of individual segments for turnover. When signed, Operations has accepted completion of the specific segment, with Design Authority concurrence. The final signatures at the end of this index reflect Operations acceptance of all operational, maintenance, and administrative responsibilities for the structures, systems, and components associated with this ABU.

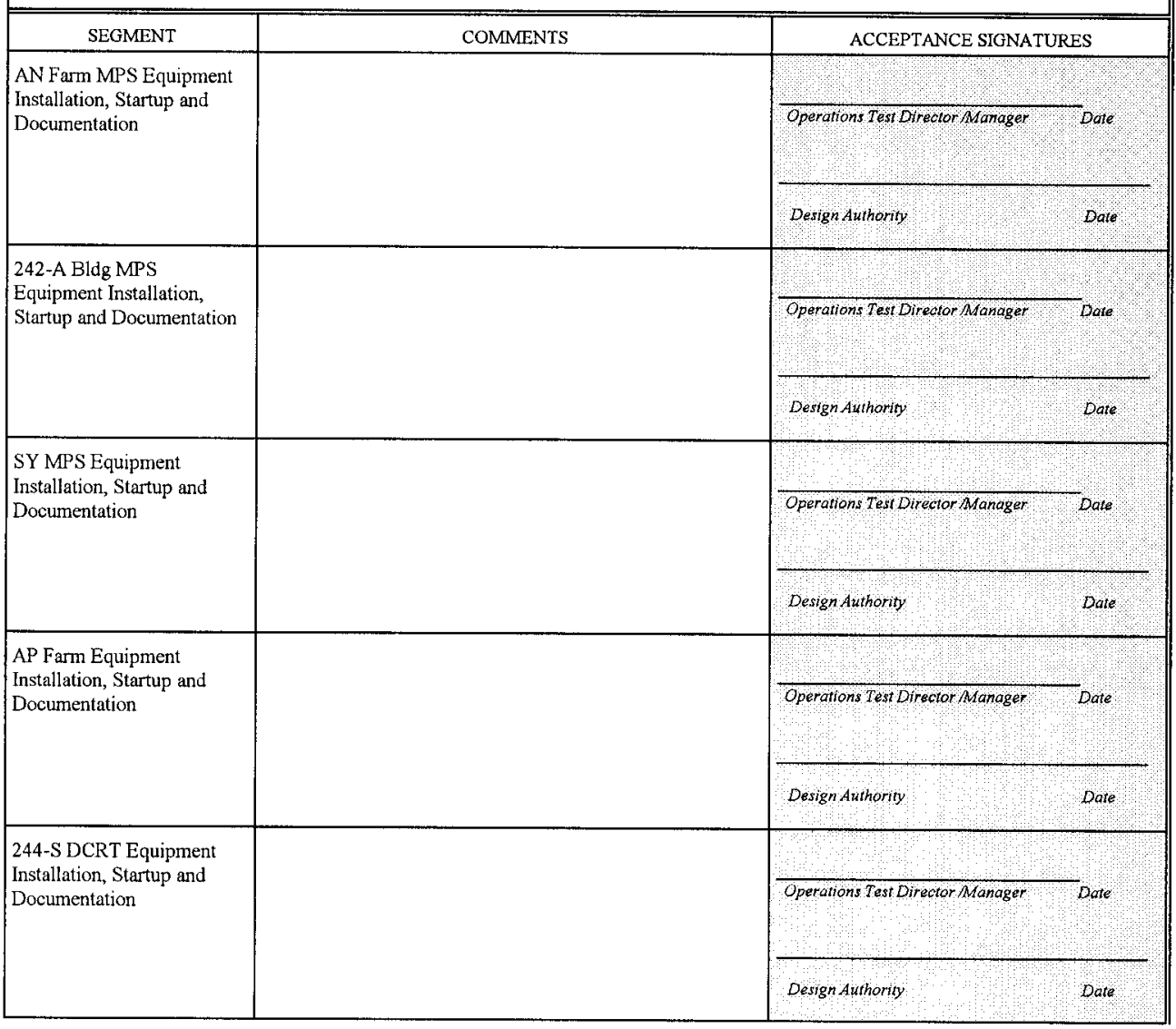


Program/Project Title: Project W-314, Tank Farm Restoration and Safe Operation, Phase 1 Component/System: Master Pump Shutdown Upgrade

September 22, 1997

AY/AZ Farms MPS

Equipment Installation, Startup and Documentation

A-Farm MPS Equipment Installation, Startup and

Documentation

(2)

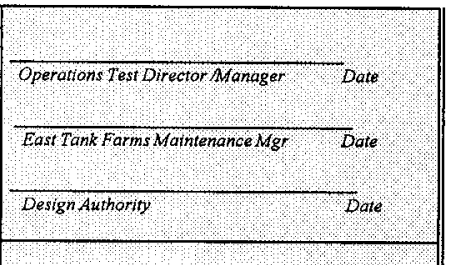

244-A DCRT MPS

Equipment Installation,

Startup and Documentation

Operallons Test Direotor Al anager Dote

Destgn Authorty $\mathrm{Dote}$

278-WA Bldg MPS

Equipment Installation,

Startup and Documentation

Operations Test Director Managar 1 Date:

272-AW Bldg MPS

Equipment Installation,

Startup and Documentation

\begin{tabular}{|l} 
\\
\hline AW-Farm MPS Equipment
\end{tabular}

Installation, Startup and

Documentation

Operations Test Director Alanoger

Pate

Design Authorty

Date 
Program/Project Title: Project W-314, Tank Farm Restoration and Safe Operation, Phase 1 Component/System: Master Pump Shutdown Upgrade

September 22, 1997

FINAL ACCEPTANCE SIGNATURES

ABU-001 for Master Pump

Shutdown Upgrades
All deliverables under "Supporting Ducument Number and Title above are complete, signed, and acceptable. Tank Farm Operations accepts full responsibility for systems, structures, and components covered by this ABU.
Operations Project Engineer

Date

Design Authority Date 
Program/Project Title: Project W-314, Tank Farm Restoration and Safe Operation, Phase 1

Component/System: Master Pump Shutdown Upgrade September 22, 1997

\section{ABU NNDEX - Post Acceptance Punch List}

This section of the index identifies items still requiring completion or closure after acceptance of the segment/system per the ABU.

Note: Items affecting Readiness may not be on the punchlist without documented justification, an approved plan to correct the deficiency, and written concurrence from the Director of Tank Farms (or his delegee).

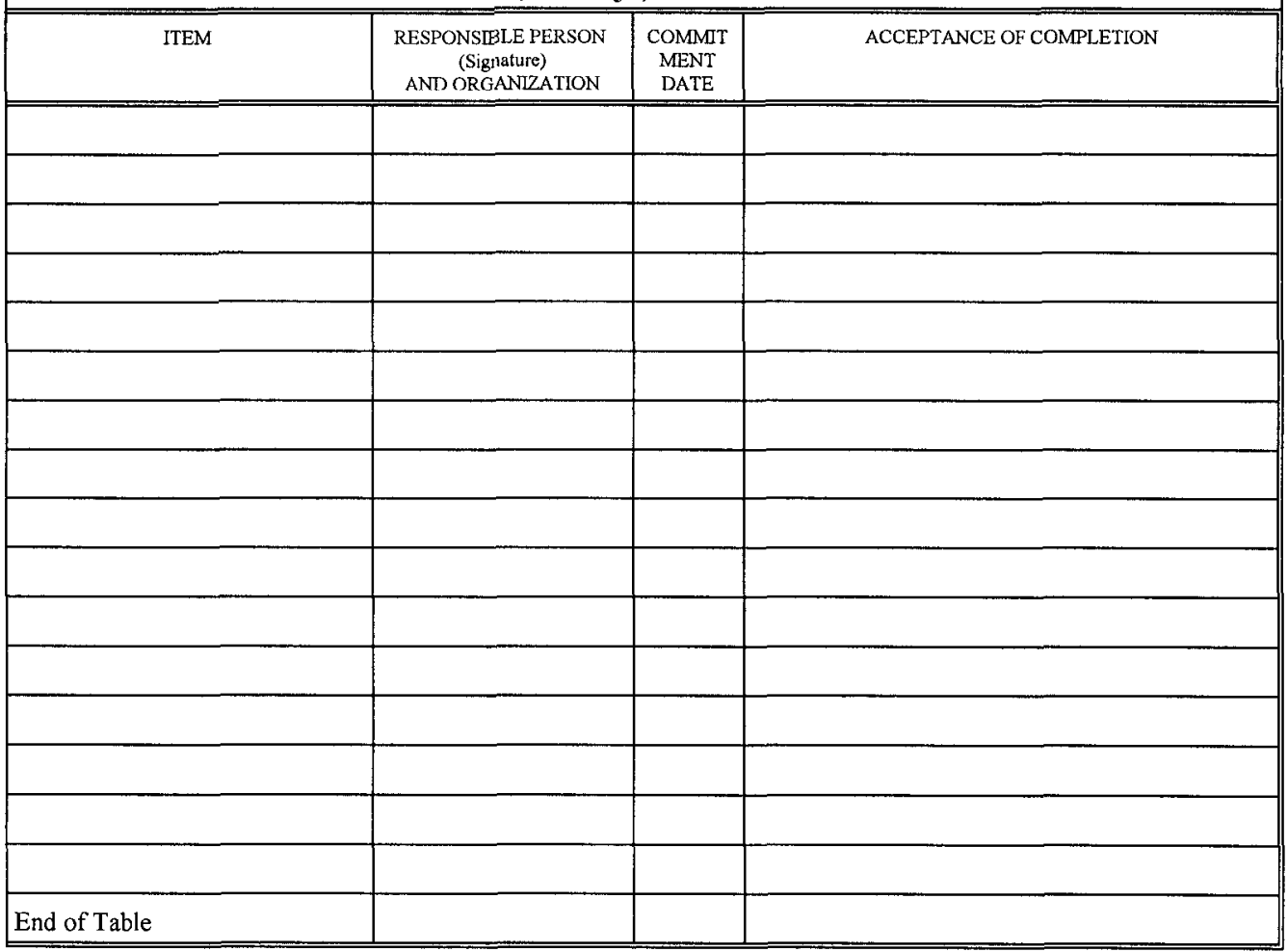




\section{DISTRIBUTION COVERSHEET}

Author

Document No.

Terry Warnick E6-21

EDT 618922

Subject: HNF-SD-W314-ABU-002

Project $W-314$

MASTER PUMP SHUTDOWN Upgrade

Acceptance for Beneficial Use

\section{DISTRIBUTION}

\begin{tabular}{|c|c|c|}
\hline Name & Location & w/att \\
\hline D. E. Bowers & $55-13$ & $x$ \\
\hline D. G. Baide & $55-05$ & $x$ \\
\hline W. M. Harty, Jr & $S 5-13$ & $\hat{x}$ \\
\hline D. Alison & $\$ 5.03$ & $\hat{x}$ \\
\hline T. Ravencraft & s5-01 & $\hat{x}$ \\
\hline R. W. Jacobson & R1-09 & $x$ \\
\hline W. E. Ross & S5.07 & $x$ \\
\hline W. W. Rutherford & $R 3-25$ & $x$ \\
\hline T. A. Erickson & $R 2-50$ & $x$ \\
\hline J. T. Koberg & $G 3-12$ & $x$ \\
\hline W. H. Hays & $E 6.08$ & $x$ \\
\hline H. M. Chaf in & $R 3-25$ & $x$ \\
\hline A. F. Erhart & $R 2-50$ & $\hat{x}$ \\
\hline J. D. McDonald & $R 2-50$ & $x$ \\
\hline R. L. Nelson & R3-47 & $x$ \\
\hline J. G. Burton & $T 4-07$ & $x$ \\
\hline C. E. Leach & R1-49 & $x$ \\
\hline J. L. Homan & $R 1-43$ & $x$ \\
\hline M. D. Rickenbach & $G 3-12$ & $x$ \\
\hline K. A. Boes & $R 3-25$ & $x$ \\
\hline D. R. Miller & G3-12 & $x$ \\
\hline J. M. Morris & $R 2-87$ & $x$ \\
\hline T. M. Horner & $\mathrm{R} 2-82$ & $x$ \\
\hline J. W. Comer & $\mathrm{R} 2 \cdot 88$ & $x$ \\
\hline D. L. McGrew & $R 3.25$ & $x$ \\
\hline W. H. Bryant & $50-09$ & $x$ \\
\hline R. J. Shupe & $R 2-50$ & $x$ \\
\hline B. L. Syverson & H5-57 & $x$ \\
\hline
\end{tabular}

Name

Location

w/att

D. E. Bai $x$

$x$

$5-13$

$1-09$

R3-47

Location w/at

H5 -57

* Advanced Distribution 